\title{
Scorpion Cardiotoxicity
}

\author{
Hilary Denis Solomons* \\ Department of Med Hematology, Pathology, University of the Witwatersrand, South Africa
}

*Corresponding author: Hilary Denis Solomons, Department of Med Hematology,

Pathology, University of the Witwatersrand, South Africa.

Received Date: June 18, 2019

Published Date: June 21, 2019

\section{Cardiotoxic Effects of Parabuthus; the South African Scorpion}

A range of cardiotoxic abnormalities occur in about one third to one half of patients with systemic envenomation, These effects include atrial tachycardia, ventricular extrasystoles, T-wave inversions, ST-T wave changes and less frequently, bundle branch block. Increased autonomic stimulation caused by increased vagal effects on the heart and sympathetic stimulation are the probable cause of these effects. Hypertension is common and occurs early in response to sympathetic stimulation. Hypotension is less common, occurs with the development of severe envenomation, and often requires intervention with vasopressors and fluid resuscitation. Many factors are at play in the development of hypotension, with cholinergic stimulation causing vasodilatation, fluid loss and myocardial depression. Cardiac dysfunction resulting from catecholamine-induced myocarditis and myocardial ischaemia complicates severe envenomation. This complication may result in pulmonary oedema and cardiogenic shock!

In cases of severe envenomation ECG, cardiac enzymes and echocardiography may be of value. This will be guided by the degree of envenomation and the facilities available. Dobutamine treats cardiogenic shock and decreases in cardiac output resulting from elevated catecholamines and myocardial injury. Nitroglycerine acts as a vasodilator for treatment of pulmonary oedema; decreases preload and afterload through arteriolar dilatation and venodilation. Atropine may also be used. Scorpion stings occur worldwide and are of clinical importance as they may cause local effects or more systemic neurotoxic and cardiovascular toxic effects. The specific treatment is antivenom combined with symptomatic and supportive treatment, including prazosin and dobutamine in patients with cardiovascular effects and benzodiazepines in neuromuscular involvement. The stinger of the scorpion releases many toxins (mostly alpha toxins.)

These toxins act on potassium and calcium channels, but these toxins appear to be less important in human envenomation!

\section{Acknowledgment}

None

\section{Conflict of Interest}

No conflict of interest. 